\title{
Efficacy Study on Interactive Mixed Reality (IMR) Software with Sepsis Prevention Medical Education
}

\author{
Naveen Kumar Sankaran ${ }^{1}$, Harris J.Nisar ${ }^{2}$, Ji Zhang ${ }^{3}$, Kyle Formella ${ }^{2}$, Jennifer Amos, PhD ${ }^{4}$, \\ Lisa T. Barker, MD ${ }^{5}$, John A. Vozenilek, MD $^{6}$, Steven M. LaValle, $\mathrm{PhD}^{7}$, Thenkurussi Kesavadas, $\mathrm{PhD}^{1}$ \\ ${ }^{1}$ Industrial and Enterprise Systems Engineering, Health Care Engineering System Center(HCESC), \\ University of Illinois at Urbana-Champaign(UIUC), Urbana, IL, USA. \\ 2Jump Trading Simulation and Education Center, OSF HealthCare System, Peoria, IL, USA. \\ ${ }^{3}$ College of Media, HCESC, UIUC, Urbana, IL, USA. \\ ${ }^{4}$ Department of Bioengineering, UIUC, Urbana, IL, USA. \\ ${ }^{5}$ University of Illinois College of Medicine at Peoria/ OSF St. Francis Medical Center, \\ OSF HealthCare System, Jump Trading Simulation and Education Center, Peoria, IL, USA. \\ ${ }^{6}$ Jump Trading Simulation and Education Center, University of Illinois College of Medicine at Peoria/ \\ OSF St. Francis Medical Center, OSF HealthCare System, Peoria, IL, USA. \\ ${ }^{7}$ Information Technology and Electrical Engineering, University of Oulu, Finland.
}

\begin{abstract}
Objective: In recent years, the training of novice medical professionals with simulated environments such as virtual reality (VR) and augmented reality (AR) has increased dramatically. However, the usability of these technologies is limited due to the complexity involved in creating the clinical content. To be comparable to a clinical environment, the simulation platform should include real-world learning parameters such as patient physiology, emotions, and clinical team behaviors. Incorporating such nondeterministic parameters has historically required faculty to possess advanced programming skills. Lack of effective software for instructors to easily develop VR curriculum content is a hurdle in developing VR based curriculum. Method: We address this challenge through a software platform that simplifies the creation of Interactive Mixed Reality (IMR) scenarios. Three educational components we were able to embed into an IMR scenario includes 1) integrated 360-degree video recording of a clinical encounter to provide a first-person perspective, 2) rich annotated knowledge content, and 3) assessment questionnaire. We developed a sepsis prevention education scenario using the IMR software to demonstrate the potential of enhancing simulated medical training by accelerating clinical exposure for novice students. Result: An IRB approved study was conducted with a group of 28 novice students to evaluate the efficacy of the IMR technology. The participants provided feedback by answering demographics, NASA-TLX and system usability scale questionnaires. Significance: Our software is a step towards improving VR based education content development
\end{abstract}

*Corresponding author email: nsankrn2@illinois.edu

1 (Email: nsankrn2, kesh @illinois.edu)

2 (Email: Harris.J.Nisar@osfhealthcare.org, kyle.t.formella@jumpsimula tion.org)

3 (Email: jizhang2@illinois.edu)

4 (Email: jamos@illinois.edu)

5 (Email: lisa.t.barker@jumpsimulation.org)

${ }^{6}$ (Email: john.a.vozenilek@jumpsimulation.org)

7 (Email: steven.lavalle@oulu.fi) process. Conclusion: The studies conducted here provide preliminary evidence that the IMR software is a usable technology based on the NASA-TLX and system usability studies conducted. Future work will compare our new educational strategy for medical training with live simulation scenarios inside a hospital room and a simple video-based curriculum.

Index Terms: $\quad$ Mixed reality-Medical training-Medical simulation—_-Sepsis training_-Emergency medicine.

\section{INTRODUCTION}

The emergence of virtual reality and augmented reality has gained tremendous attention in both academia and industry. To create those environments, one must model the content of the scenario in a process much like that used to computer graphics in movies. Currently, neither VR nor AR can be used easily to create an extensive simulated medical curriculum that can demonstrate clinical and surgical procedures. Milgram et al. [13] proposed a 'Reality Virtuality Continuum,' and introduced 'Mixed Reality (MR)' to cover various combinations of AR \& VR technologies. In the Reality Virtuality Continuum, MR represents all simulated environments in which the real world and virtual world are juxtaposed. MR applications can be developed to provide viewers with immersive experiences that have both real and virtual content, thereby relaxing the intense modelling requirement for creators.

In our innovative approach, instead of completely replicating the real world scenario in VR, we propose to represent the realworld as prerecorded information (360-degree panoramic videos) in Mixed Reality. The prerecorded scenarios along with overlaid graphic content, are delivered in an Interactive Mixed Reality (IMR) experience for the viewers. The prerecorded IMR provides extensive functions, such as navigation within the scenario, rich annotation content (text, images, video demos, 3D models, etc.), andassessment tools for learning. Our proposed IMR strategy is used to create medical content for novice medical professionals in specific content areas.

Clinical education and training are a complex knowledge domain that requires rigorous educational processes and skill development training. Conventional medical education strategies include textbook learning, didactics, practical lab sessions, and workplace training [9]. Years of didactics and continuous workplace training precede qualification for clinical practice [11]. Moreover, conventional medical training has the disadvantages of being expensive and 
time-consuming. In addition, in the clinical environment, learners cannot practice at will, and the inherent convenience sampling of clinical cases may limit educational opportunities. In the apprenticeship model, the learning happens over a period of time, and it takes several hundred cases or procedures before a level of advanced competency is reached. Bridges et al. [3] estimated a cost of \$53 million per year to train American residents in operating rooms, which includes facility usage and training time. A study on training of surgical residents for basic competency in robotic surgery calculated that it costs $\$ 672,000$ to train 105 residents inside an operating room using animals. However, when a simulator was used, the cost was reduced to around $\$ 125,000$ [15]. This significant cost difference is one indication of how medical education can benefit from advanced simulators.

Simulation-based training aims to reproduce real medical situations for students and has been demonstrated to improve learning outcomes. Larsen et al. [10] reported that VR simulations used in laparoscopy training can reduce operation times, while resulting in higher proficiency among trainees. Similar outcomes in other areas of medical simulation training have been presented in related studies $[1,7,17,18]$. For some procedures, such as robotic surgery training, simulators such as RoSS have been shown to be very effective $[4,14]$.

A curriculum intended for novice medical professionals must include well-defined learning objectives or tasks at an appropriate level of difficulty with focused, repetitive practice [12]. To engage learners, it is appealing to create a platform as immersive as the clinical environment [6]. To achieve such high fidelity, the virtual platform should include real-world learning parameters to prompt the user for information on the tasks required of the targeted learner.

Multiple strategies could be used for such simulation technologies. VR can provide a good training environment [2], but developing VR scenarios is extremely time-consuming and tedious. AR applications can extend the usability of medical simulation training by providing an extended workspace and interaction with the real world. Carolien et al. [9] discuss multiple AR medical training applications, such as human anatomy visualization and laparoscopic skill training. Despite its advantages in overlaying information on the current real-world, its usability to provide broad training content is limited due to the complexity involved in creating the AR application. Alternatively, the proposed MR strategy promises new possibilities for developing simulated medical scenarios. For our MR application, prerecorded real medical scenarios were embedded and overlaid with graphical content to enhance the immersive experience. The platform successfully reproduces details of hospital routines and practices. Trainees can observe the actions, movements, and behavior of the demonstrating experts as well as the patient. The trainee can also interact with objects and get real-time feedback on their actions.

In this project we tested the usability of our proposed IMR application by creating a module for sepsis management. Sepsis is a medical condition that results from the human body's inflammatory response to infection, which can lead to tremendous physiological derangement. Without prompt diagnosis and treatment, the condition can rapidly develop into septic shock, organ failure, and death. Sepsis is very common around the world, and almost one million cases are recorded every year in the United States [8]. Should sepsis progress to septic shock, patients become highly dependent on intensive care and continuous medical support. Thus, training for novice medical professionals is foundational to creating awareness about sepsis in hospital and clinical environments. To test the efficacy of the IMR technology, we used a sepsis prevention scenario is used as content to get user feedback (according to an IRB protocol) from a group of volunteers.

This paper is organized as follows Section II provides analysis of the simulation requirements involved in creating a sepsis scenario.
Section III explains the components of IMR in developing a sepsis scenario. Section IV describes the design of our study for evaluating the software's efficacy. Section V \& VI present our results and a discussion of our sepsis prevention training scenario and the user study data.

\section{Methods}

\subsection{Sepsis Task Analysis for Interactive Mixed Reality}

Understanding the components of a sepsis case is essential in developing effective Interactive Mixed Reality content on sepsis. The fundamental phases of the tasks involved in emergency situations are situational assessment, decision making, task selections and sequencing, and task activities [5]. Addressing of those fundamental tasks guides the proper structure of IMR scenarios that address emergency medicine cases such as sepsis.

\subsubsection{Situational Assessment}

When an emergency case is presented to a care team, immediate attention and response is required to address derangements in the patient's physiology. In a clinical setting, this may occur in a setting of extreme cognitive demand, and it is crucial for staff to assess the patient's condition in a speedy and accurate manner. An assessment begins with a briefing from paramedics or nurses followed by examination (visual, verbal, and physical) of the patient's status. Simulation training content on that process for novice must be immersive, engaging, and cognitively demanding, much like an actual hospital. The IMR software integrates realistic scenarios that support the learning and participation required for a situational assessment based on clinical knowledge.

\subsubsection{Decision-making}

The knowledge and skills required for medical decision-making are gradually being taught to students [16]. An expert medical care provider's decision-making skills are the result of complex thought processes that are difficult to explain elaborately during a clinical event (when the patient demands immediate attention). For example, an expert's decision to administer a drug to a patient is an outcome of the experts analysis of the patient's vital signs and medical history combined with an understanding of drug responses and side effects. The complex information processing in the expert's mind involves recollection, understanding, and analysis that must be effectively presented to the novice during training. Thus, during IMR curriculum development, care providers must be able to provide annotations that discuss complex thought processes and decisionmaking outcomes.

\subsubsection{Task Selection and Sequencing}

Based on a patient's condition, the clinical care team may need to repeatedly switch tasks to stabilize the patient's condition. Introductory training and exposure to task scheduling and teamwork in an ER can be provided to novices in the IMR context.

\subsubsection{Task Activities Learning}

Training novices to learn task activities includes teaching them the knowledge behind the task, the procedures involved in the task, and the sensorimotor skills required to perform the task. These activities for sepsis scenarios have been identified as intravenous access, culture sample collection, and the cleaning and drilling required for intravenous access. The IMR curriculum will provide basic introductory training to the novices on these tasks. It is not intended to replace the standard hands-on training; the goal is to provide a simulation-based introduction to the importance of team activity in safety-critical tasks. The following section discusses the feasibility of developing an IMR curriculum, and addresses the identified tasks in sepsis emergency training. 


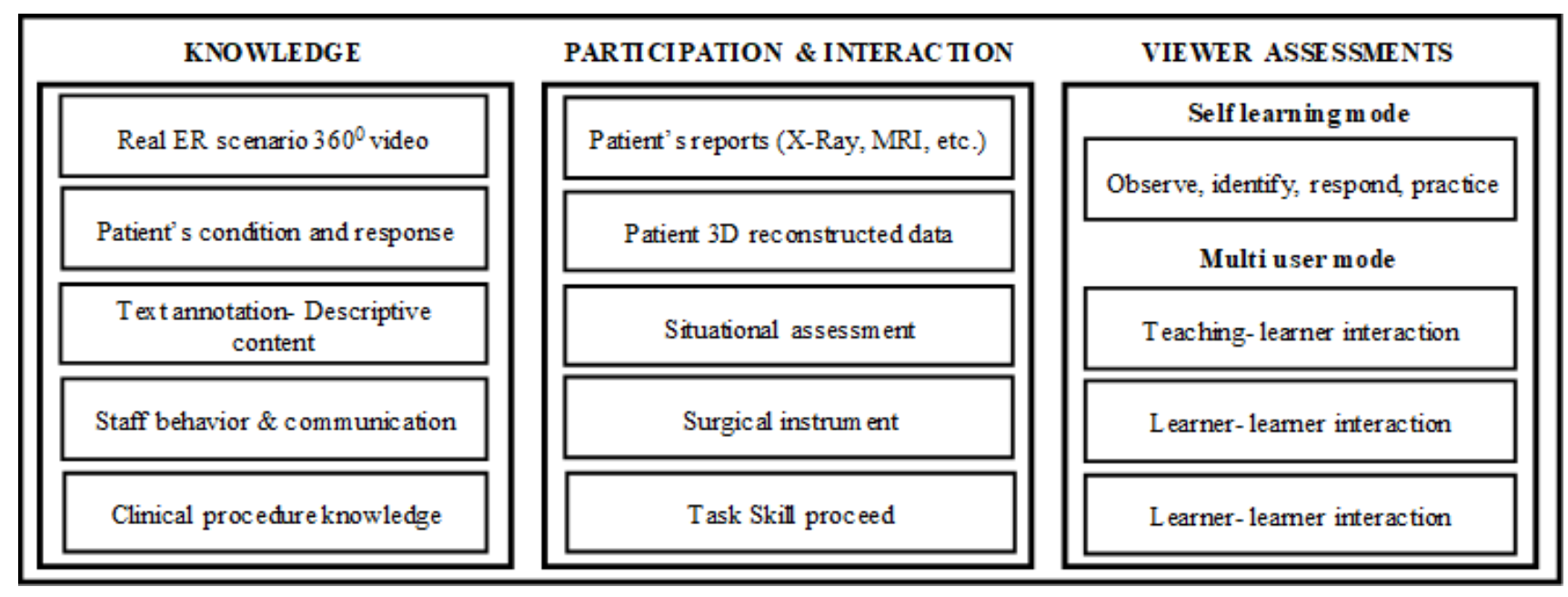

Figure 1: Component of IMR Sepsis curriculum.

\section{Serious imR Curriculum Components for Novice TRAINING}

To develop an effective sepsis training curriculum using IMR, it is essential to address the challenges identified in the previous section. The components of the proposed IMR curriculum are shown in Fig 1. The primary components that provide situational awareness in IMR curriculum ensure accurate representation of real-world scenarios without complicating the curriculum development itself. we addressed those challenges by recording a 360-degree video of an expert clinician responding to a real reenacted ER sepsis case. The recorded real-world 360-degree video playback eases IMR curriculum development by eliminating the need to model a graphical representation of fine details of the ER. Moreover, the viewer is provided with a first-person view, ensuring immersion inside the ER. Thus, the situational awareness training is provided via presentation of the playback video. The participation and situational assessment of the viewer are ensured via interactions in the IMR.

The knowledge components of our IMR curriculum are provided by rich annotation content (text, images, 3D models, videos, etc.) of standard learning material overlaid on top of playback video. The annotation feature also facilitates to annotate about staff member's complex thought and decision-making processes. During IMR curriculum development, the staff can provide rich visual content to support elaborate discussions about treatment and expected outcomes.

Viewer participation in our IMR curriculum is ensured with goaldriven activities, engaging questions, and timely feedback at appropriate stages of the scenario, as determined by the clinical context. Examples of interactive content provided in the IMR includes handling of (i) patients' reports; (ii) patients' physiological data, including imaging and electronic medical reports; and (iii) medical devices user interface. Other modalities, including 3D reconstruction of patient models, can also be provided where appropriate. Further, learners will be required to perform task sequencing for the given task in the scenario. The responses to the questions can be used for assessment and also to provide reasoning with annotation, if the wrong sequences of tasks are performed. It has also been proposed that one can teach a task procedure by presenting the trainee with a demonstration of a task execution in a video or 3D animations. Examples of tasks that could be taught that way include ECG lead placement and central venous catheter placement.

Trainee assessment in the IMR curriculum has been developed to provide both self-learning and multi-user modes. In the self-learning mode, the trainee is guided and assessed by the IMR software. In the multi-user mode, the curricula accommodate multiple users who can participate in a scene simultaneously facilitating live interactions among trainees, instructors, and simulation technicians. That provides an opportunity for content discussion and team activities during the training session.

The sepsis module development began with recording of a 360degree video of a sepsis encounter. Fig. 2 shows a stretched-out view of the 360-degree scenario. When viewed with a head-mounted display, it gives viewers a first-person view of the scenario happening in the patient's room. Via our software, the 360-degree video has been embedded with rich annotated content. Fig. 3 shows an example of embedded knowledge content about sepsis in the 360-degree video. It also shows interactions of the trainee with the contents in the curriculum using standard VR controller. Enhanced visual representations of real-world information can be provided to the viewer in the IMR. Fig. 4 shows an enhanced graphical representation of a patient's vitals.

The serious training in the IMR application is achieved by embedding assessment questionnaire in the scenario. Fig. 5. shows a sample standard AVPU question that is being presented to the viewer. The viewers response can be recorded to provide constructive feedback to help the learning process.

\section{Design of a Study for Evaluating Software Effi- CACY}

The IMR software is used to create education content by integrating 360-degree videos, numerous multimedia features and assessment. To test the efficacy of the IMR technology, we used the sepsis prevention scenario as content to get user feedback about the software, following IRB protocols. The goal of this study is to obtain feedback on application (Sepsis prevention education) built using the IMR software. In the future, this software will be commercialized as product to easily create variety of mixed reality education content. The participants in the trial consisted of 28 Bioengineering students from the University of Illinois at Urbana-Champaign. (Note: The scenario sepsis prevention education developed with our software is introductory level medical education. The participant population represented educational background that satisfies premedical education requirement.) The students had an average age of $19.03 \pm 0.18$ years, and $18.25 \%$ of the students were males, while $81.75 \%$ were female.

The participants wore a head-mounted display (HTC Vive), held a handheld controller, and played the sepsis-prevention content created with our IMR software. As the objective of the study is to evaluate 


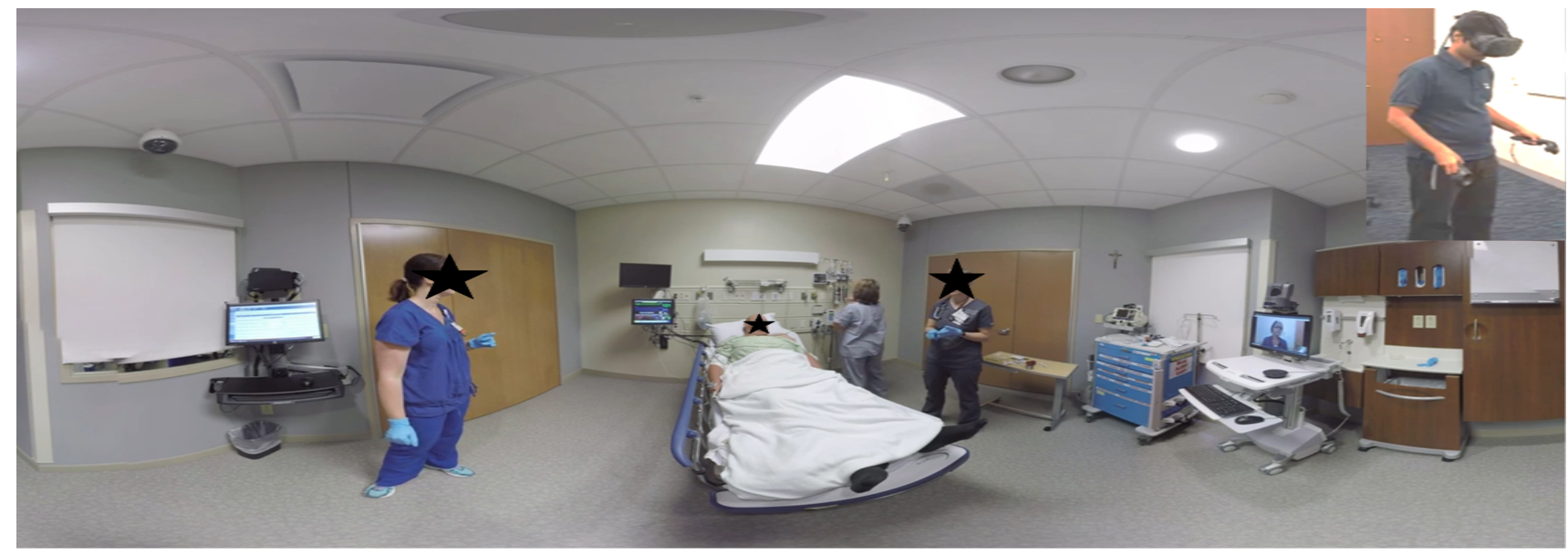

Figure 2: Stretched-out screenshot of the 360-degree video of the sepsis training IMR module. The scene shows the overall environment of the room, with the patient in the middle and the physicians discussing the patient's condition.

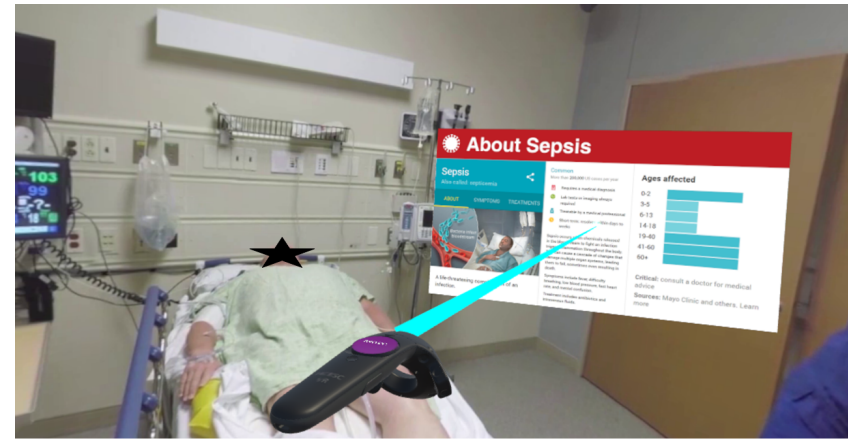

Figure 3: Screenshot of the user's first-person view of the sepsis training application with rich educational pop-up information explaining the sepsis condition.

the efficacy of the software a short version (3mins 30sec) of the sepsis education is presented to the participants instead of full version (21mins). The short version highlights the critical scenes during the training along with features of interactions and multimedia and assessments. The full version is intended for comprehensive leaning as part of curriculum. For this study, the participants were asked to use the IMR application and evaluate its performance. Throughout the study, participants were trained to use the IMR application along with the handheld controller and to perform all the experiments (Note: The participants responses to assessments activities inside the sepsis education were not evaluated during this study. Future studies will be planned to evaluate participants leaning experience using IMR applications.)

\section{Pre-study questionnaires}

Eligibility: There are no target populations. Anyone can participate, since the technology is designed to be used by all, regardless of sex, race, or age. In order to ensure subjects safety, eligibility criteria were set based on the HTC Vive safety information guide. The subjects were required to complete a pre-study eligibility questionnaire and only eligible subjects were allowed to participate in the study. In addition, each participant was asked to read the HTC Vive safety information guide consent form (English version), and was required to provide consent, with the date, at the bottom of each page. The eligibility criteria questionnaires are included in the Appendix section Fig. 9 for reference.

Demographics: We collected demographic details about the participants' experience with mixed reality technologies. The questionnaire captured participants' programming experience, VR program-

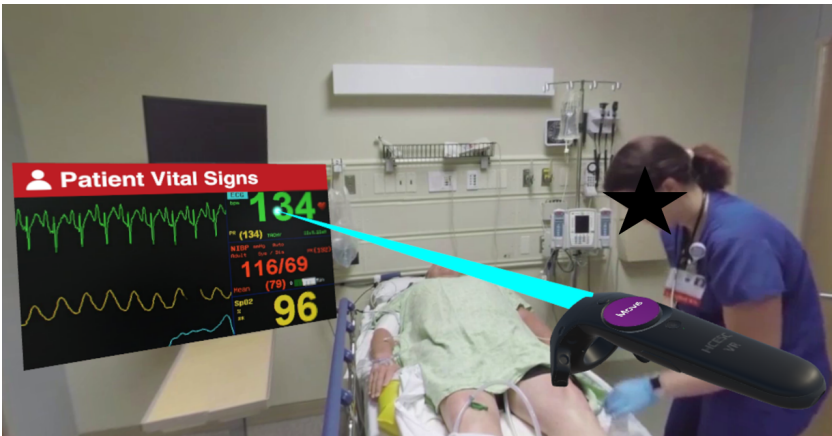

Figure 4: Screenshot of the user's first-person view of the sepsis training application, with an enhanced graphic showing the patient's vital signs for better observation and emphasis.

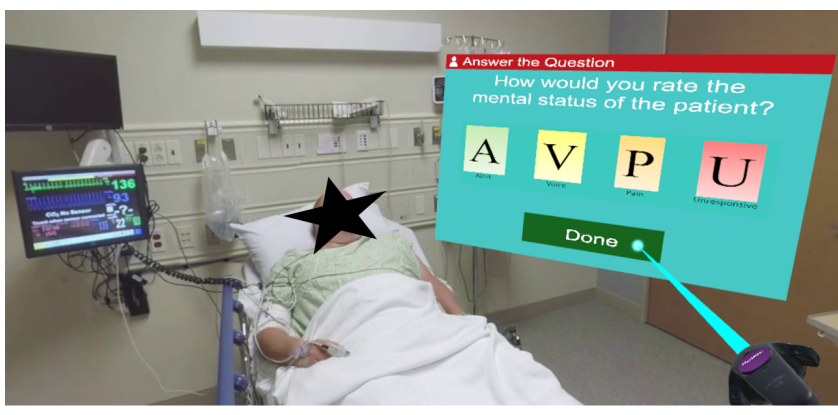

Figure 5: Screenshot of the user's first-person view of the sepsis training application, with a real-time questionnaire to test the user's learning progress.

ming experience, gaming experience, VR gaming experience, and experience watching 360-degree videos.

\section{Post-study questionnaires:}

NASA-TLX: We used this to evaluate the mental, physical, and temporal demands, frustration, effort, and performance of, the participants in using the IMR application. The NASA-TLX questionnaire is a subjective workload assessment technique that derives an overall workload score based on six weighted subclasses with a range of 0 (low) to 20 (high).

System Usability Scale: We used this scale to evaluate the interaction feature of the IMR application. It was used to evaluate the overall usability of the module based on a ten-item, Likert-scale 


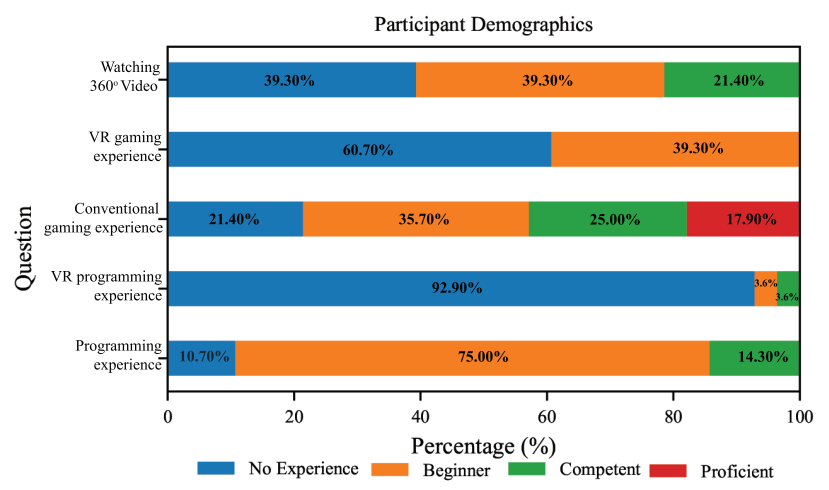

Figure 6: Participant demographics.

questionnaire. Each response could range from 1 (strongly disagree) to 5 (strongly agree).

Next we discuss the efficacy of the IMR software, based on the study.

\section{Results}

\subsection{Demographics:}

The results of the demographics questionnaire are presented in Fig. 6 . The majority of the participants were beginners in programming $(75.0 \%)$, while $14.3 \%$ were competent, and the rest $(10.7 \%)$ had no experience. Furthermore, the majority of participants had no experience in VR programming $(92.90 \%) ; 3.4 \%$ were beginners, and $3.4 \%$ were competent in VR programming. In terms of conventional gaming experience, the participants' distribution was the most diverse, with $35.7 \%$ beginners, $25.0 \%$ competent, $21.4 \%$ no experience, and $17.9 \%$ proficient. All participants had no experience $(60.7 \%)$ or were beginners $(39.3 \%)$ in regard to VR gaming, with the majority saying they had no experience. In terms of watching 360 -degree videos, $39.3 \%$ had no experience, $39.3 \%$ were beginners, and $21.4 \%$ were competent.

\subsection{NASA-TLX:}

The results of the NASA-TLX questions are presented in Fig. 7. The scores, ranging from 0 to 20 , are grouped into three categories: Low (score $\leq 6)$, Medium $(6<$ score $\leq 13)$, and High (score $>13$ ). We consolidated all the participants' scores for each subclass by computing averages and the standard deviation. We categorized the scores into groups of Low, Medium, and High following the one-way T-test (with a $95 \%$ confidence interval). The performance score was High $(15.0 \pm 3.21 ; p<<0.05)$, while mental demand (7.04 $\pm 3.26 ; p<<0.0001)$, physical demand $(4.26 \pm 2.93 ; p<<$ $0.0001)$, temporal demand $(6.27 \pm 3.49 ; p<<0.0001)$, frustration level $(6.08 \pm 4.32 ; p<<0.0001)$, and effort $(7.65 \pm 3.91 ; p<<$ $0.0001)$ were not High. In addition to being categorized as not High, physical demand was also categorized as Low $(p<<0.05)$.

\subsection{System Usability Scale:}

We consolidated the results of the system usability questionnaire as averages and the standard deviation. Those numbers and the distribution of the scores are presented in Fig. 8. When asked if the system was relaxing, $76 \%$ of the participants gave a score of 4 or 5 , with an overall average score of $3.84 \pm 1.10$. $80 \%$ of the participants gave a score of 4 or 5 , with an overall average score of $4.04 \pm 0.97$. In regard to whether the system functions were well-integrated, $92 \%$ of participants gave a score of 4 or 5 , with an overall average score of $4.24 \pm 0.72$. When asked if people would learn to use the system quickly, $80 \%$ of participants gave a score of 4 or 5 , with an overall average score of $4.04 \pm 0.88$. In terms of how confident the participants felt while using the system, $56 \%$ of

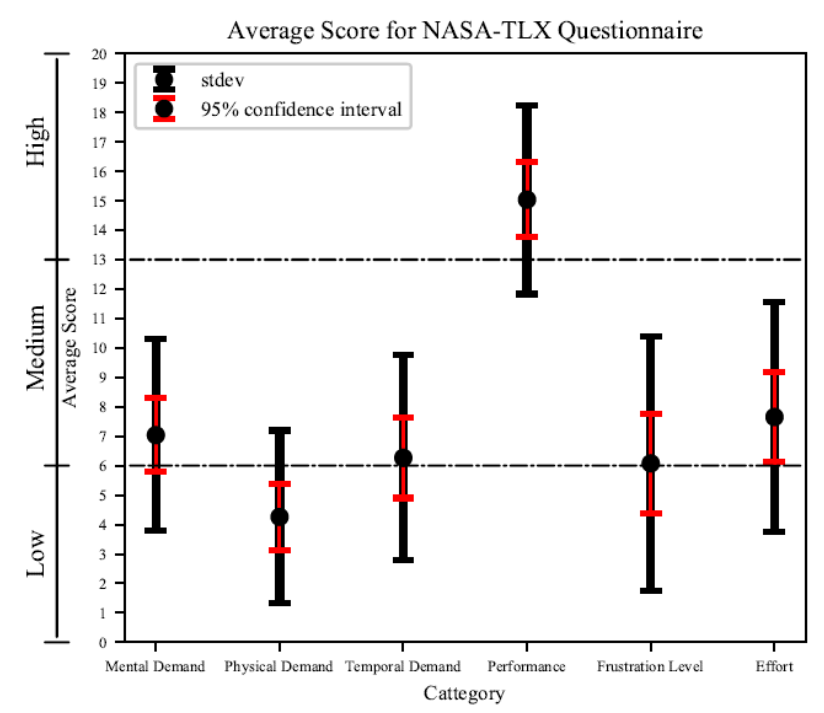

Figure 7: NASA-TLX questions.

the participants gave a score of 4 or 5 , with an overall average score of $3.52 \pm 1.32$. In regard to whether the system was unnecessarily complex, $76 \%$ of participants gave a score of 1 or 2 with an average score of $1.92 \pm 1.15$. When asked if the participants felt like they needed the support of a technical person to use the system, $28 \%$ gave a score of 1 or 2 , and $64 \%$ gave a score of 3 or 4 , with an overall average of $3.04 \pm 1.2$. In terms of how inconsistent the system was, $76 \%$ of participants gave a score of 1 or 2 with an overall average score of $1.92 \pm 0.95$. In regards to if the system was cumbersome to use, $76 \%$ of participants gave a score of 1 or 2 with an overall average score of $2.12 \pm 0.92$. When asked if the participants needed to learn a lot of new things before using the system, $68 \%$ of participants gave a score of 2 or 3 with an average of $2.76 \pm 1.01$.

\section{DISCUSSION}

\subsection{NASA-TLX:}

The NASA-TLX scores presented in Figure 7 represent the cognitive demand imposed by the IMR learning modality. The imposed mental demand is categorized as a medium mental demand. That means the mental workload required by the system is in an acceptable range; any user can learn from the IMR application without experiencing high mental demand. The physical demand is categorized as Low, implying that the IMR software is easy to use physically (that is the controller and user interfaces are easy to use). The temporal demand is categorized as not High, as the learning content can be paused by the user. Further, the frustration level is categorized as not High, implying that the IMR software did not cause high levels of frustration to users interacting with various integrated functions. Similarly, Effort is also not High as participants felt they did not have to put a large amount of effort into using the software. The performance was categorized as High, implying that the participants felt that the system performed well in delivering content. Those results are encouraging, as the software performed with a high score range and the mental demand, temporal demand, frustration level, and effort are all categorized as Low or Medium score range.

Furthermore, the NASA-TLX results can also be interpreted along with knowledge of the demographic data(Fig. 6) to understand the full impact of the IMR software's efficacy. The majority of participants in this study were not familiar with critical aspects of mixed reality (e.g. they had no experience with VR gaming or watching 360-degree videos) before experiencing IMR. Eventhough 

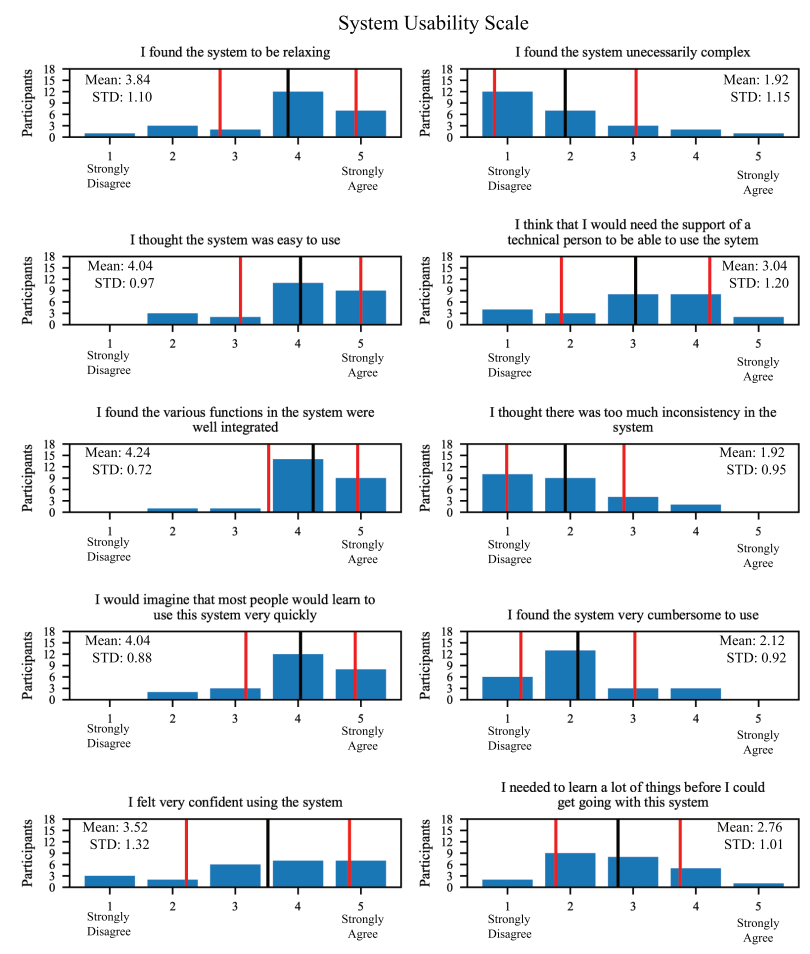

Figure 8: System usability scale responses

we had a large population of participants who were inexperienced in mixed reality, the mental, physical, temporal demands, and effort were Low or Medium. Alternatively, this tool was presented as a teaching modality to the participant (Bioengineering student who were novice to understand medical procedures), and provided them with an opportunity to learn about sepsis prevention. This clarifies the reason for the result of Low or Medium range score for mental demand, temporal demand, frustration level, and effort. Furthermore, although the majority of the participants either had no experience or were beginners in programming and/or VR programming, the performance of the software was ranked as High. That confirms that the IMR software performed well without demanding high technical skills in programming and that it is easy to use for any user. This cognitive workload in exchange for high performance is imperative in a successful learning experience.

\subsection{System Usability Scale:}

The system usability scale results presented in Figure 8 indicate that the IMR technology is usable. In our discussion of the results, scores 4 and 5 are grouped as 'Agreeing'; scores 1 and 2 are grouped as 'Disagreeing'; and score 3 is considered as 'Neutral' with respect to the question asked. The majority of the participants agreed that the IMR application is relaxing. They did not feel anxious or tense while using it. The functions were well-integrated in the system, according to the majority of participants. The participants did not feel that any of the features of the system were out of place, and the experience felt coordinated. Most of the participants agreed that they would learn to use the system quickly, as it was easy to understand and all the features and functions were well-explained. When asked how confident the participant felt while using the system, the majority of participants agreed that they were confident. Although only $56 \%$ of the participants felt confident, it is clear from the demographics that large population of the participants were experiencing IMR for the first time, as they had had limited or no experience in mixed related technologies (Fig. 6). The majority of participants agreed that the system was not unnecessarily complex. The system is easy to use, and all parts of the experience were easy to access and navigate through. When asked whether they needed a technical support person in order to use the technology, the majority of participants were neutral or agreed. Again, the feeling that technical support was needed can be attributed to the participants' lack of experience with IMR technology. The majority of participants said that the system was not inconsistent or cumbersome to use. All interactions felt how they should have, and there was little to no variance between them. Finally, when asked if they felt they needed to learn a lot of new things before using the system, the majority of participants gave a score of 2,3 , or 4 . This implies that the majority of participants felt they needed additional information to use the system, which may again reflect their lack of MR experience.

Overall, the IMR software received positive responses from the system usability study. Even though the participants were not familiar with VR or MR technologies as evident by the demographic data, the software was usable for the participants of this study. All users' feedback will be taken into consideration during later phases of the IMR technology development. While most of the feedback was positive, there was negative feedback directly related to participants, familiarity with VR and MR technology.

\section{Conclusion}

Enhancement of simulated medical training with an IMR curriculum can accelerate clinical exposure for novice students in the comfort of a classroom or home. Prerecorded real-world scenarios, along with rich annotations of content and interactions, provide immersive learning experiences to the user while reinforcing pathophysiology principles. The studies conducted here provide preliminary evidence that the IMR software is a usable technology based on the NASA-TLX and system usability studies conducted. Validation of the sepsis IMR module will be performed with a response study that includes novice medical students(currently pending). We expect to demonstrate this new model of medical training is at least as good as reenactment of scenarios inside a hospital room. Further, there is significant savings for the simulation center, since the multiple sessions of the module may be presented in a virtual environment if the program is to be presented throughout the year. In our preliminary analysis, $\$ 32,000$ savings have been projected. Further, there may be additional cost avoidance by saving transportation time to visit the Simulation center. The new environment may also offer additional learning benefits that will be studied in more detail in future work.

\section{ACKNOWLEDGMENTS}

We would like to thank the Jump ARCHES Endowment through the UIUC Health Care Engineering Systems Center and the Jump Simulation Center at OSF Health Care for supporting this project. The project could not have been completed without the contributions of the following students: Matthew K.Ong, Yingying Ren, Andrew Hehner Miranti, Nicholas Alexander Magerko, Saleh Raghib Muhammad, and Tian Menglin.

\section{REFERENCES}

[1] R. Bharathan, S. Vali, T. Setchell, T. Miskry, A. Darzi, and R. Aggarwal. Psychomotor skills and cognitive load training on a virtual reality laparoscopic simulator for tubal surgery is effective. European Journal of Obstetrics \& Gynecology and Reproductive Biology, 169(2):347352,2013

[2] J. D. Bric, D. C. Lumbard, M. J. Frelich, and J. C. Gould. Current state of virtual reality simulation in robotic surgery training: a review. Surgical endoscopy, 30(6):2169-2178, 2016.

[3] M. Bridges and D. L. Diamond. The financial impact of teaching surgical residents in the operating room. The American Journal of Surgery, 177(1):28-32, 1999. 
[4] A. Chowriappa, S. J. Raza, A. Fazili, E. Field, C. Malito, D. Samarasekera, Y. Shi, K. Ahmed, G. Wilding, J. Kaouk, et al. Augmented-realitybased skills training for robot-assisted urethrovesical anastomosis: a multi-institutional randomised controlled trial. BJU international, 115(2):336-345, 2015.

[5] S. de Ribaupierre, B. Kapralos, F. Haji, E. Stroulia, A. Dubrowski, and R. Eagleson. Healthcare training enhancement through virtual reality and serious games. In Virtual, Augmented Reality and Serious Games for Healthcare 1, pp. 9-27. Springer, 2014.

[6] D. M. Gaba. The future vision of simulation in health care. Quality and safety in Health care, 13(suppl 1):i2-i10, 2004.

[7] T. P. Grantcharov, V. Kristiansen, J. Bendix, L. Bardram, J. Rosenberg, and P. Funch-Jensen. Randomized clinical trial of virtual reality simulation for laparoscopic skills training. British Journal of Surgery, 91(2):146-150, 2004.

[8] I. Jawad, I. Lukšić, and S. B. Rafnsson. Assessing available information on the burden of sepsis: global estimates of incidence, prevalence and mortality. Journal of global health, 2(1), 2012.

[9] C. Kamphuis, E. Barsom, M. Schijven, and N. Christoph. Augmented reality in medical education? Perspectives on medical education, 3(4):300, 2014.

[10] C. R. Larsen, J. Oestergaard, B. S. Ottesen, and J. L. Soerensen. The efficacy of virtual reality simulation training in laparoscopy: a systematic review of randomized trials. Acta obstetricia et gynecologica Scandinavica, 91(9): 1015-1028, 2012.

[11] M. LLC. Acgme common program requirements.

[12] W. C. McGaghie, S. B. Issenberg, M. E. R. Cohen, J. H. Barsuk, and D. B. Wayne. Does simulation-based medical education with deliberate practice yield better results than traditional clinical education? a metaanalytic comparative review of the evidence. Academic medicine: journal of the Association of American Medical Colleges, 86(6):706, 2011

[13] P. Milgram, H. Takemura, A. Utsumi, and F. Kishino. Augmented reality: A class of displays on the reality-virtuality continuum. In Pho tonics for industrial applications, pp. 282-292. International Society for Optics and Photonics, 1995.

[14] S. J. Raza, S. Froghi, A. Chowriappa, K. Ahmed, E. Field, A. P. Stegemann, S. Rehman, M. Sharif, Y. Shi, G. E. Wilding, et al. Construct validation of the key components of fundamental skills of robotic surgery (fsrs) curriculuma multi-institution prospective study. Journal of surgical education, 71(3):316-324, 2014

[15] S. Rehman, S. J. Raza, A. P. Stegemann, K. Zeeck, R. Din, A. Llewellyn, L. Dio, M. Trznadel, Y. W. Seo, A. J. Chowriappa, et al. Simulation-based robot-assisted surgical training: a health economic evaluation. International Journal of Surgery, 11(9):841-846, 2013
[16] J. C. Rogers, D. E. Swee, and J. A. Ullian. Teaching medical decision making and students' clinical problem solving skills. Medical teacher, 13(2):157-164, 1991

[17] B. Schout, H. J. Ananias, B. L. Bemelmans, F. C. dAncona, A. M. Muijtjens, V. E. Dolmans, A. J. Scherpbier, and A. J. Hendrikx. Transfer of cysto-urethroscopy skills from a virtual-reality simulator to the operating room: a randomized controlled trial. BJU international, 106(2):226-231, 2010.

[18] H. W. Schreuder, G. Oei, M. Maas, J. C. Borleffs, and M. P. Schijven. Implementation of simulation in surgical practice: minimally invasive surgery has taken the lead: the dutch experience. Medical teacher, 33(2):105-115, 2011

\section{ApPEndiX}

\section{Pre-Study Eligibility Questiomaire}

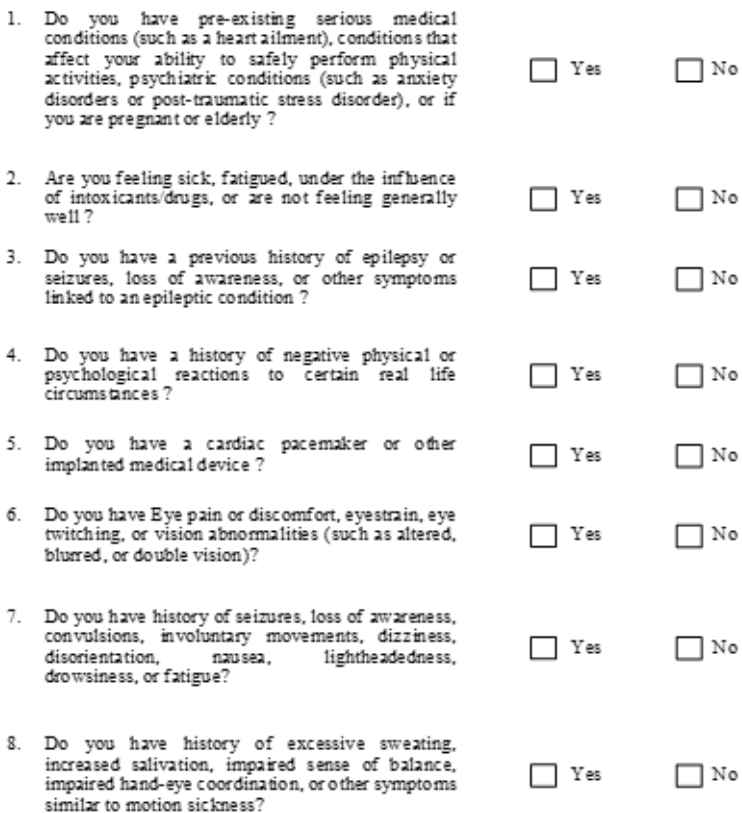

Figure 9: Eligibility criteria questionnaire. 